\title{
Electronic transport through Si nanowires: Role of bulk and surface disorder
}

\author{
Markussen, Troels; Rurali, R.; Brandbyge, Mads; Jauho, Antti-Pekka
}

Published in:

Physical Review B Condensed Matter

Link to article, DOI:

10.1103/PhysRevB.74.245313

Publication date:

2006

Document Version

Publisher's PDF, also known as Version of record

Link back to DTU Orbit

Citation (APA):

Markussen, T., Rurali, R., Brandbyge, M., \& Jauho, A-P. (2006). Electronic transport through Si nanowires: Role of bulk and surface disorder. Physical Review B Condensed Matter, 74(24), 245313.

https://doi.org/10.1103/PhysRevB.74.245313

\section{General rights}

Copyright and moral rights for the publications made accessible in the public portal are retained by the authors and/or other copyright owners and it is a condition of accessing publications that users recognise and abide by the legal requirements associated with these rights.

- Users may download and print one copy of any publication from the public portal for the purpose of private study or research.

- You may not further distribute the material or use it for any profit-making activity or commercial gain

- You may freely distribute the URL identifying the publication in the public portal 


\title{
Electronic transport through Si nanowires: Role of bulk and surface disorder
}

\author{
Troels Markussen, ${ }^{1}$ Riccardo Rurali, ${ }^{2}$ Mads Brandbyge, ${ }^{1}$ and Antti-Pekka Jauho ${ }^{1}$ \\ ${ }^{1}$ MIC, Department of Micro and Nanotechnology, NanoDTU, Technical University of Denmark, DK-2800 Kgs. Lyngby, Denmark \\ ${ }^{2}$ Departament d'Enginyeria Electrònica, Universitat Autònoma de Barcelona 08193 Bellaterra, Spain \\ (Received 13 June 2006; revised manuscript received 15 September 2006; published 15 December 2006)
}

\begin{abstract}
We calculate the resistance and mean free path in long metallic and semiconducting silicon nanowires (SiNW's) using two different numerical approaches: a real-space Kubo method and a recursive Green'sfunction method. We compare the two approaches and find that they are complementary: depending on the situation a preferable method can be identified. Several numerical results are presented to illustrate the relative merits of the two methods. Our calculations of relaxed atomic structures and their conductance properties are based on density functional theory without introducing adjustable parameters. Two specific models of disorder are considered: Unpassivated, surface reconstructed SiNW's are perturbed by random on-site (Anderson) disorder whereas defects in hydrogen passivated wires are introduced by randomly removed $\mathrm{H}$ atoms. The unpassivated wires are very sensitive to disorder in the surface whereas bulk disorder has almost no influence. For the passivated wires, the scattering by the hydrogen vacancies is strongly energy dependent and for relatively long SiNW's $(L>200 \mathrm{~nm})$ the resistance changes from the Ohmic to the localization regime within a $0.1-\mathrm{eV}$ shift of the Fermi energy. This high sensitivity might be used for sensor applications.
\end{abstract}

DOI: 10.1103/PhysRevB.74.245313

PACS number(s): 73.63.-b, 72.15.Lh, 72.10.Fk

\section{INTRODUCTION}

Semiconducting nanowires are a very promising building block for future nanoelectronic and nanophotonic applications as witnessed by several recently demonstrated devices. ${ }^{1-6}$ Silicon nanowires (SiNW's) are especially attractive candidates due to their compatibility with conventional $\mathrm{Si}$ technology and due to the accurate control of diameter and electronic properties during synthesis. ${ }^{7}$ Furthermore, in recent years SiNW's have been applied as label-free realtime chemical and biological sensors with very high sensitivity and, e.g., capability of single-virus detection. ${ }^{8}$

Thin SiNW's with diameters below $5 \mathrm{~nm}$ have been synthesized by several groups. Recently, Ma et al. ${ }^{9}$ obtained very thin wires grown in the $\langle 110\rangle$ and $\langle 112\rangle$ directions with diameters down to $1.3 \mathrm{~nm}$ and Holmes et al. ${ }^{10}$ previously reported 4-5-nm $\langle 100\rangle$ and $\langle 110\rangle$ SiNW's. Wu et al. ${ }^{7}$ recently demonstrated that the growth directions depend on the diameter, which can be controlled by the size of a catalytic nanoparticle. ${ }^{11}$

Concerning theoretical modeling of the structural properties, Rurali and Lorente ${ }^{12}$ recently showed, using $a b$ initio calculations, that thin $\langle 100\rangle$ unpassivated SiNW's could be either metallic or semimetallic depending on the surface reconstruction. In another recent work, Singh et al. ${ }^{13}$ theoretically studied pristine $\langle 110\rangle$ SiNW's and found that these were indirect band gap semiconductors. Fernández-Serra $e t$ $a l .{ }^{14}$ used ab initio calculations to study the surface segregation of dopants in both passivated and unpassivated SiNW's, and Vo et al. ${ }^{15}$ very recently used $a b$ initio calculations to simulate the structural and electronic properties of hydrogenpassivated SiNW's grown in different directions.

The large surface to bulk ratio in the thin wires implies that surface effects such as defects, vacancies, or adatoms will have a large influence on the transport properties. This was experimentally demonstrated by Cui et al. ${ }^{4}$ showing increased mobilities after passivation of surface defects. Also, electron-phonon scattering might be suppressed in thin wires. Indeed, recent experiments by Lu et al. ${ }^{16}$ indicated ballistic transport in undoped $\mathrm{Si} / \mathrm{Ge}$ core-shell wires at room temperature with an estimated phonon scattering mean free path (MFP) $l_{p h}>500 \mathrm{~nm}$. This might imply that even at room temperature defects could be the most important scattering source, and a thorough understanding of the scattering processes is thus required.

A number of transport calculations have been reported for wires with various diameters. Das and Mizel ${ }^{17}$ used the Boltzmann equation to calculate the carrier mobility in relative thick $(d=10-90 \mathrm{~nm})$ GaAs wires, focusing on the diameter dependence. Sundaram and Mizel ${ }^{18}$ also used the Boltzmann equation to study surface effects on the transport in largediameter wires. Zheng et al. ${ }^{19}$ applied a tight-binding model of a hydrogen-passivated wire and studied the effect of wire thickness on the band gap, effective masses, and transmission.

Real SiNW's with lengths up to the scale of micrometers consist of millions of atoms and are likely to have many randomly placed defects. To our knowledge, no theoretical works concerning SiNW's, based on ab initio methods and including many scattering events, have yet been published.

A calculation of the conductance of a SiNW with many randomly positioned defects puts strong requirements on the method. The quasi-one-dimensional nature of the SiNW's requires on the one hand an atomistic model taking quantum effects and charge transfer around the defect into account. On the other hand, the method should be able to treat more than $10^{5}$ atoms and include many scattering events due to the $\mu \mathrm{m}$ length of the wires.

In this work, two methods are used to study the effect of disorder including many randomly placed $\mathrm{H}$ vacancies in H-passivated long SiNW's. Both methods are based on $a b$ initio calculations and scale linearly with the length of the sample. The first approach uses a relatively recent real-space method developed by Roche and Mayou and co-workers 
over the last decade to study transport properties primarily in carbon nanotubes and quasiperiodic systems. ${ }^{20-28}$ The method is based on the Kubo-Greenwood formalism ${ }^{29,30}$ rewritten in a real-space framework, and we will refer to it as the Kubo method. The second and more well-known approach is based on the Landauer formula where the conductance is found by recursive calculations of Green's functions (GF's). We will refer to this as the GF method.

The Kubo method was shown to predict the elastic MFP at the Fermi energy in randomly disordered carbon nanotubes (CNT's) ${ }^{24}$ in agreement with a Fermi's golden rule estimate. ${ }^{31}$ Besides that, we are aware of no comparison with other theoretical methods. One of the primary goals of this paper is to report such a comparison over several energies. We show that the Kubo and GF methods are in general in qualitative agreement, however with quantitative differences especially around band edges. We analyze the pros and cons of the two methods and give an assessment of when they should be applied and which quantities they can calculate.

The rest of the paper is organized as follows. In Sec. II we summarize the two numerical methods and describe how a Hamiltonian for a long SiNW is constructed from ab initio calculations. Results concerning both unpassivated as well as hydrogen-passivated SiNW's are presented in Sec. III. We end up with a discussion of the applied methods and the results in Sec. IV.

\section{METHODS}

\section{A. Real-space Kubo method}

In the real-space Kubo method, which is derived from the Kubo-Greenwood formula, transport properties are determined by calculating the time propagation of wave packets in real space. The central quantity is the time- and energydependent diffusion coefficient $\mathcal{D}(E, t)$, defined by

$$
\mathcal{D}(E, t)=\frac{1}{t} \frac{\operatorname{Tr}\left\{[\mathbf{X}(t)-\mathbf{X}(0)]^{2} \delta(E-\mathbf{H})\right\}}{\operatorname{Tr}\{\delta(E-\mathbf{H})\}},
$$

where $\mathbf{X}(t)$ is the position operator along the wire direction written in the Heisenberg representation, $\mathbf{H}$ is the Hamiltonian matrix, and the trace $\operatorname{Tr}\{\delta(E-\mathbf{H})\}$ is the total electronic density of states (DOS). Since the Hamiltonian in the calculations is finite, the energy has a small imaginary part $i \eta$, where $\eta \sim 5 \mathrm{meV}$ is chosen to scale linearly with the total band width and inversely with the system size. Following Triozon et al. ${ }^{24,32}$ an efficient evaluation of the traces can be carried out by using a relative modest number $(<10)$ of random phase states $\left|\psi_{r}\right\rangle$. The coefficients of $\left|\psi_{r}\right\rangle, \psi_{r}(j)$, with $j=-N / 2, \ldots, N / 2$ where $N$ is the total number of orbitals in the wire, are initially nonzero only in the central part of the nanowire-i.e.,

$$
\psi_{r}(j)=\left\{\begin{array}{cc}
\frac{1}{\sqrt{N_{r}} e^{2 i \pi \alpha_{r}(j)},} & \text { for }-N_{r} / 2 \leqslant j \leqslant N_{r} / 2, \\
0 & \text { otherwise, }
\end{array}\right.
$$

where $\alpha_{r}(j)$ is an independent random number in the interval $[0,1]$ for every $(r, j)$. Initially, $\left|\psi_{r}(t=0)\right\rangle$ is located in the

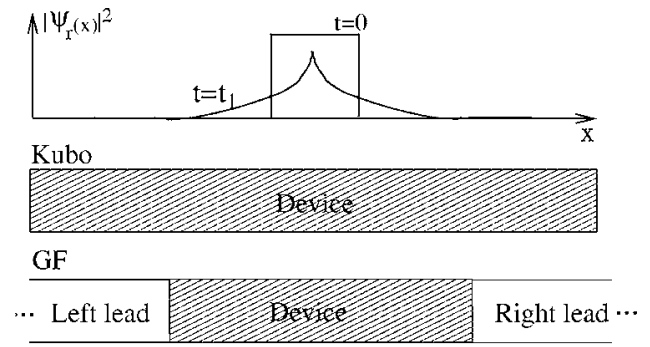

FIG. 1. Top: schematic time evolution of a random phase state $\left|\psi_{r}(t)\right\rangle$ initially located in the central region of the wire. Middle: the geometry in the Kubo method consists only of a large device region. Bottom: in the GF approach a device region is connected to two semi-infinite leads.

central part of the wire, and as time evolves it spreads out to the sides as illustrated in Fig. 1 (top). In order to avoid scattering at the boundaries at large propagation times, the initial range of $\left|\psi_{r}\right\rangle$ must be much smaller than the total system. Typically $N_{r} \sim 10^{4}$ while the total number of orbitals is $N$ $\sim 10^{5}$. The number of random phase states needed to accurately estimate the traces is not known a priori, and the convergence of the results must be checked.

The time evolution of the random phase states can be efficiently computed by expanding the time evolution operator $e^{-i \mathbf{H} t / \hbar}$ in the orthogonal set of Chebyshev polynomials. Each term in the traces in Eq. (1) is a local density of state which is calculated using a continued fraction technique. ${ }^{33}$ This is the most time-consuming part of the calculations and involves $\sim 10^{3}$ operations with the Hamiltonian for the considered systems to resolve the closely lying energy bands. The convergence of the continued fraction scheme must be separately verified.

The elastic $\operatorname{MFP} l_{e}\left(E_{F}\right)$ for a given energy $E=E_{F}$ is found from $^{24}$

$$
l_{e}\left(E_{F}\right)=\frac{\max \left\{\mathcal{D}\left(E_{F}, t\right), t>0\right\}}{v\left(E_{F}\right)},
$$

where $v(E)$ is an energy-dependent effective velocity. For a pristine system the electron motion is ballistic and the diffusion coefficient increases linearly with time, $\mathcal{D}(E, t)=v^{2}(E) t$, with the slope given by the square of the effective velocity. In Sec. III A and the Appendix we discuss this effective velocity in more detail. We note that in the ballistic regime $l_{e}$ $\rightarrow \infty$ and the "max" in Eq. (3) is not well defined.

The geometry used in the Kubo calculations is sketched in Fig. 1 (middle). Notice that there is no requirement that the Hamiltonian be periodic. Nor are there any leads that connect to the device region as in the GF method, Fig. 1 (bottom), to be discussed in the next section.

We stress that the time it takes to calculate the diffusion coefficient at many energies is not much longer than for a single energy. The reason for this is that the random phase states contain all energy components and the time evolution is energy independent. Moreover, the primary numerical task in the continued fraction scheme is a mapping of the original Hamiltonian to a smaller tridiagonal matrix. This mapping is 


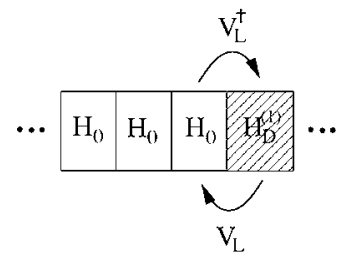

$\mathrm{L}$

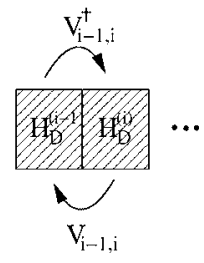

D



$\mathrm{R}$
FIG. 2. The device region $(D)$ is divided into $M$ subcells. The subcells are so large that they only couple to the nearest-neighbor cells. Also, the left $(L)$ and right $(R)$ leads consisting of equal unit cells, described by $\mathbf{H}_{0}$, couple only to the first and last cell of the device region, respectively.

energy independent, and it is therefore relatively fast to compute the MFP for the whole energy spectrum.

\section{B. Recursive Green's function method}

The second numerical method we have applied is based on the Landauer formalism described in detail in, e.g., Ref. 34. The general setup is illustrated in Fig. 2. A device region $(D)$ is connected to a left $(L)$ and right $(R)$ semi-infinite lead. The device area consists of $M$ subcells and is described by the Hamiltonian $\mathbf{H}_{D}$ :

$$
\mathbf{H}_{D}=\left(\begin{array}{cccc}
\mathbf{H}_{D}^{(1)} & \mathbf{V}_{1,2} & 0 & \ldots \\
\mathbf{V}_{1,2}^{\dagger} & \mathbf{H}_{D}^{(2)} & \mathbf{V}_{2,3} & \\
0 & \mathbf{V}_{2,3}^{\dagger} & \ddots & \ddots \\
\vdots & & \ddots & \mathbf{H}_{D}^{(M)}
\end{array}\right) .
$$

The subcells are chosen so large that only nearest-neighbor cells couple. Generally, the subcells do not need to have the same size. The leads described by $\mathbf{H}_{L}$ and $\mathbf{H}_{R}$, respectively, are assumed to have a semi-infinite structure consisting of equal unit cells with Hamiltonians $\mathbf{H}_{0}$. The coupling matrices between the leads and the device area are denoted $\mathbf{V}_{L}$ and $\mathbf{V}_{R}$. The Hamiltonians are calculated with a nonorthogonal basis set (see Sec. II C) such that for each Hamiltonian matrix we also have a corresponding overlap matrix $\mathbf{S}$.

To calculate the length dependent conductance of the wire, we initially find the surface Green's functions $\mathbf{G}_{L, R}^{0}(E)=\left(E \mathbf{S}_{L, R}-\mathbf{H}_{L, R}\right)^{-1}$ of the isolated leads by a standard decimation procedure. ${ }^{35}$ The device area is subsequently grown by adding one subcell at a time and calculating the Green's function

$$
\mathbf{G}_{i}(E)=\left[E \mathbf{S}_{D}^{(i)}-\mathbf{H}_{D}^{(i)}-\Sigma_{L}^{(i)}(E)-\Sigma_{R}(E)\right]^{-1},
$$

where $\Sigma_{R}(E)$ describes the coupling to the right lead and is defined through $\mathbf{G}_{R}^{0}(E)=\left[E \mathbf{S}_{0}-\mathbf{H}_{0}-\Sigma_{R}(E)\right]^{-1}$, and where we assure that the coupling of cell $i$ to lead $R$ is the same as the cell-to-cell coupling within the lead. $\Sigma_{L}^{(i)}(E)$ also takes the coupling to the rest of the device area into account and is calculated from the previous growth step as $\Sigma_{L}^{(i)}(E)$ $=\left(E \mathbf{S}_{i-1, i}^{\dagger}-\mathbf{V}_{i-1, i}^{\dagger}\right) \widetilde{\mathbf{G}}_{i-1}(E)\left(E \mathbf{S}_{i-1, i}-\mathbf{V}_{i-1, i}\right)$, where $\quad \widetilde{\mathbf{G}}_{i-1}(E)$ $=\left[E \mathbf{S}_{D}^{(i-1)}-\mathbf{H}_{D}^{(i-1)}-\Sigma_{L}^{(i-1)}(E)\right]^{-1}$.

In each growth step we calculate the conductance of the wire consisting of the first $i$ subcells as

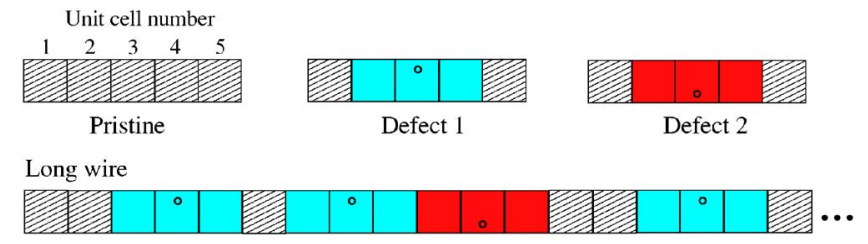

FIG. 3. (Color online) Top: schematic illustration of supercells containing five unit cells from three different DFT calculations: one pristine wire and two wires with different defects. In the latter the defects are located in unit cell number 3 . The cells number 1 and 5 are assumed to be the same in all three calculations. Bottom: a long wire is constructed by joining pieces from the different DFT calculations.

$$
g\left(E, L_{i}\right)=\frac{2 e^{2}}{h} \operatorname{Tr}\left[\mathbf{G}_{i}^{\dagger}(E) \Gamma_{R}(E) \mathbf{G}_{i}(E) \Gamma_{L}^{(i)}(E)\right],
$$

where $L_{i}$ is the length of the grown device region, $\Gamma_{L}^{(i)}(E)=$ $-2 \operatorname{Im}\left[\Sigma_{L}^{(i)}(E)\right]$, and likewise for $\Gamma_{R}(E)$. The trace is performed over the states in the device region.

Sample averaging is performed over 200 different configurations, giving a mean conductance $\langle g\rangle$. The corresponding resistance is found as $R=1 /\langle g\rangle$. For wire length $L \ll \xi$, with $\xi$ being the localization length, the resistance increases linearly as $R(L)=R_{0}+R_{0} L / l_{e}$, defining the MFP $l_{e} \cdot{ }^{36}$ In the localization regime the resistance increases exponentially and we calculate the localization length as ${ }^{37}$

$$
\xi=-\lim _{L \rightarrow \infty} \frac{2 L}{\langle\ln g\rangle},
$$

where $L$ has to be so large that $\xi$ is converged.

\section{Constructing the Hamiltonian from $a b$ initio calculations}

The atomic and electronic structure of the SiNW's is found from $a b$ initio calculations using the density functional theory (DFT) package SIESTA. ${ }^{38}$ Using first-principles calculations it is relatively straightforward to introduce various defects such as vacancies, adatoms, or dopants. This is not the case when using standard tight-binding parameters.

We have used a minimal single- $\zeta$ basis set, ${ }^{39}$ with four orbitals (one $3 s$ and three $3 p$ ) on the $\mathrm{Si}$ atoms and one on the $\mathrm{H}$ atoms, to represent the one-electron wave function. The minimal basis set is applied in order to speed up the subsequent transport calculations. We have used norm-conserving pseudopotentials of the Troullier-Martins type ${ }^{40}$ and the generalized gradient approximation ${ }^{41}$ for the exchangecorrelation functional. The calculations are performed on supercells containing five wire unit cells (see Figs. 3 and 4). The reciprocal space has been sampled with a converged grid of $1 \times 1 \times 2 k$ points following the Monkhorst-Pack scheme. ${ }^{42}$

When modeling a defect this is placed in the middle of the supercell to ensure that the effect of the defect is confined to the central region and is not affected by the periodicity and the intercell coupling terms $V_{i-1, i}$ in Eq. (4) are independent of the specific type of defect. The atomic postions of all the atoms in the unit cell containing the defect and in the two neighboring unit cells have been fully relaxed, until the 


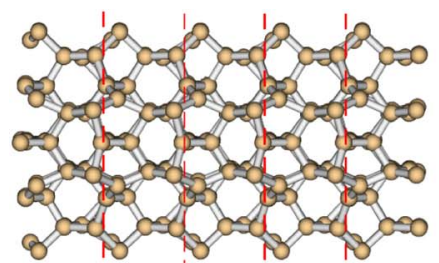

(a)

(b)

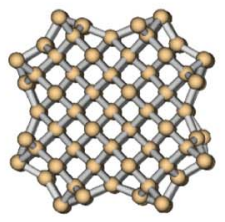

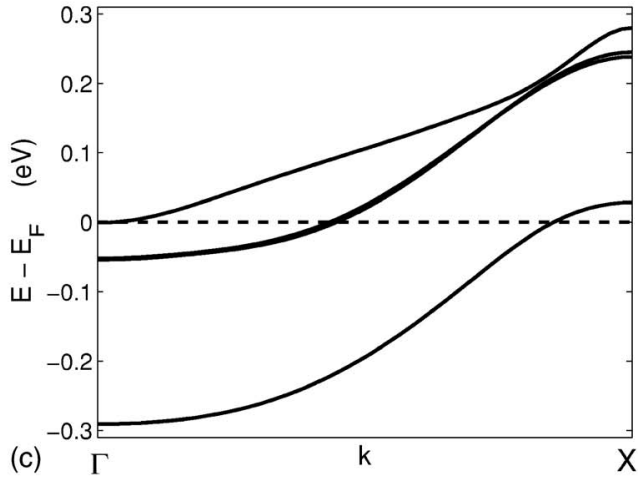

FIG. 4. (Color online) (a): Side view of the unpassivated SiNW containing five unit cells, separated by the red dashed lines. (b): End view of the unpassivated SiNW. (c): Band structure around the Fermi energy (dashed line). Four bands are crossing the Fermi energy, two being degenerate (with $E-E_{F}=-0.05 \mathrm{eV}$ at the $\Gamma$ point).

$$
\mathbf{H} \rightarrow \widetilde{\mathbf{H}}=\mathbf{S}^{-1 / 2} \mathbf{H} \mathbf{S}^{-1 / 2} .
$$

In the same way as described above, a long wire is built by extracting parts from both pristine and defected orthogonalized Hamiltonians and joining the pieces. The Löwdin transformation leads to a longer range of the basis orbitals and thus more nonzero elements in the truncated Hamiltonian. This in turn implies longer calculation times.

\section{Fermi's golden rule}

We next compare the two numerical approaches with results obtained using Fermi's golden rule (FGR). We consider scattering due to the scattering potential $V$ between unperturbed Bloch states $|n, k\rangle$ of the pristine wire. The transport relaxation rate $1 / \tau_{n}$ from band $n$ at energy $E$ is found as

$$
\begin{aligned}
\frac{1}{\tau_{n}\left(E_{n}(k)\right)}= & \frac{2 \pi}{\hbar} \int \frac{\mathrm{d} k^{\prime}}{2 \pi} \sum_{m} \overline{\left|\left\langle k^{\prime}, m|V| n, k\right\rangle\right|^{2}} \times(1 \\
& \left.-\cos \theta_{k k^{\prime}}\right) \delta\left(E_{m}\left(k^{\prime}\right)-E_{n}(k)\right) \\
= & \frac{4 \pi}{\hbar} \sum_{m} \overline{\left|\left\langle-k^{\prime \prime}, m|V| n, k\right\rangle\right|^{2}} n_{m}(E) \\
= & \frac{4 \pi}{\hbar} \sigma^{2} \sum_{m, j}\left|\psi_{-k^{\prime \prime}, m}(j)\right|^{2}\left|\psi_{k, n}(j)\right|^{2} n_{m}(E),
\end{aligned}
$$

where the $m$ summation is over bands and the $j$ summation runs over sites in one unit cell. $\psi_{k, n}(j)=\langle j \mid k, n\rangle$ is the amplitude of the Bloch state at site $j, n_{m}(E)$ is the DOS corresponding to band $m$, and the energy of the final state fulfills $E_{m}\left(-k^{\prime \prime}\right)=E_{n}(k)=E$. $\theta_{k k^{\prime}}$ is the angle between the initial and final wave vectors. Only scattering from a forward- to a backward-propagating state contributes to the rate-i.e., $\theta_{k k^{\prime}}=-\pi$-yielding a factor of 2 . The perturbing potential $V$ is for the Anderson on-site disorder (Sec. III A) simply a diagonal matrix with the diagonal elements being random numbers in a given interval with variance $\sigma^{2}$ (see below), and the overbar denotes an average over different configurations of $V$. The MFP for electrons in band $n$ is calculated as

$$
l_{n}(E)=v_{n}(E) \tau_{n}(E),
$$

where $v_{n}(E)$ is the group velocity at energy $E$ in the $n$th band. We calculate the total MFP as the mean value from the individual contributions from the bands: 


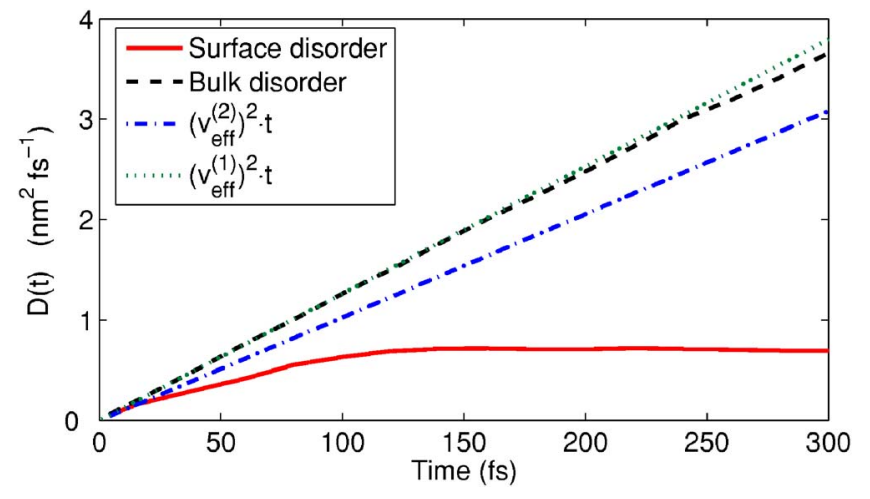

FIG. 5. (Color online) Time-dependent diffusion coefficient at $E-E_{F}=0.1 \mathrm{eV}$. The bulk disorder has little effect and the transport is ballistic. The edge-disordered system shows diffusive behavior with a constant diffusion coefficient for $t>150 \mathrm{fs}$.

$$
l_{e}(E)=\frac{1}{N(E)} \sum_{n} l_{n}(E),
$$

where $N(E)$ is the number of bands at energy $E$.

\section{RESULTS}

\section{A. Surface-reconstructed wires}

The first structures we consider are unpassivated, surface reconstructed SiNW's as illustrated in Fig. 4 showing a side view (a) and a cross-section view (b) of a wire containing 5 unit cells, separated by the red dashed lines in the upper panel. This structure was recently studied by Rurali and Lorente $^{12}$ using DFT calculations. There are 57 atoms in one unit cell, the diameter is $\sim 1.5 \mathrm{~nm}$, and the length of the unit cell in the growth direction is $0.55 \mathrm{~nm}$. Notice that the structure at the surface differs significantly from the bulk of the wire. Reference 12 showed that this particular surface structure makes the wire metallic, which is evident in Fig. 4(c), showing the band structure around the Fermi energy (marked by the dashed line). Four bands are crossing the Fermi level (two being degenerate).

To further investigate the metallic surface states and to compare the Kubo and GF methods we add random on-site noise to either the surface atoms only or to the bulk atoms only. The relevant diagonal elements in the Hamiltonian are thus changed according to

$$
\mathbf{H}_{i i} \rightarrow \mathbf{H}_{i i}+\delta_{i},
$$

where $\delta_{i}$ takes values with equal probability in the interval $[-\Delta \varepsilon / 2 ; \Delta \varepsilon / 2]$ with $\Delta \varepsilon$ being the disorder strength. The uniform disorder distribution has variance $\sigma^{2}=(\Delta \varepsilon)^{2} / 12$. This Anderson model for electronic disorder is simple and widely applied; very recently, Zhong and Stocks ${ }^{43}$ used it to model surface disorder in shell-doped nanowires. Whether this simple model adequately describes physical defects merits a separate discussion, given at the end of Sec. III B.

Figure 5 shows $\mathcal{D}(E, t)$ at $E-E_{F}=0.1 \mathrm{eV},{ }^{44}$ revealing that the bulk disorder (dashed black) has a very small effect on the transport properties, yielding an almost linearly increas- ing diffusion coefficient $\mathcal{D}(E, t)=v^{2}(E) t$, a characteristic feature of ballistic transport, as discussed in Sec. II A. The surface disorder (solid red), on the other hand, leads to diffusive transport with $\mathcal{D}(E, t)$ almost constant for $t>150$ fs. The pronounced differences of the surface- and bulk-disordered systems show that the conduction around the Fermi energy almost entirely takes place along the surface atoms, in qualitative agreement with the conclusions drawn in Ref. 12. The two additional curves show $\left(v_{\mathrm{eff}}^{(1)}\right)^{2} t$ (dotted green) and $\left(v_{\mathrm{eff}}^{(2)}\right)^{2} t$ (dash-dotted blue), where $v_{\text {eff }}^{(1)}=\sqrt{\sum_{m} n_{m} v_{m}^{2} / \Sigma_{m} n_{m}}$ $=0.112 \mathrm{~nm} / \mathrm{fs}$ and $v_{\mathrm{eff}}^{(2)}=\Sigma_{m} n_{m} v_{m} / \Sigma_{m} n_{m}=0.101 \mathrm{~nm} / \mathrm{fs}$ are calculated from the band structure, Fig. 4(c), according to the discussion in the Appendix. The band velocities are $v_{1,2}$ $=0.157 \mathrm{~nm} / \mathrm{fs}$ for the degenerate band and $v_{3}=0.059 \mathrm{~nm} / \mathrm{fs}$ for the highest-lying band. It is evident that the effective velocity calculated in the Kubo method from the slope of the linearly increasing diffusion curve is very close to $v_{\mathrm{eff}}^{(1)}$ and not $v_{\text {eff }}^{(2)}$. As shown in the Appendix this would lead to an overestimated conductance of a pristine wire when this is calculated as described in the Appendix A and Ref. 23.

Using Eq. (3) the MFP for the surface-disordered system is found to be $l_{e}=6.4 \mathrm{~nm}$. Using the velocity $v_{\mathrm{eff}}^{(1)}$ we find the mean free time $\tau=l_{e} / v_{\text {eff }}^{(1)}=57$ fs which is approximately half of the time where the diffusion curve levels off.

The total length of the SiNW's in the Kubo calculations is $L=825 \mathrm{~nm}$, corresponding to 1500 unit cells. Even though the group velocity around the Fermi energy is $\sim 0.1 \mathrm{~nm} / \mathrm{fs}$ and the wave packets thus only propagate $30 \mathrm{~nm}$ in the 300 fs shown in Fig. 5, there are higher velocities at other energies. Moreover, at other energies where the MFP is longer the propagation time must also be longer to reach the flat plateau in the diffusion curve. In order to avoid the wave packet being reflected at the ends of the wire at long propagation times, which affects the calculated diffusion, we find that very long wires are needed.

Figure 6(a) shows the MFP $l_{e}(E)$ versus energy calculated using both the Kubo method (solid blue) and the recursive GF approach (black squares). The dashed red line is an analytical estimate obtained using FGR. The disorder strength is $\Delta \varepsilon=0.4 \mathrm{eV}$ and only the surface atoms are perturbed. The Kubo and FGR results are obtained with a fine energy resolution whereas the GF results are calculated only for relatively few, discrete energies, illustrating the advantages in the Kubo method in calculating properties at many energies in one calculation.

Generally all three methods agree qualitatively and in the interval $-0.1 \mathrm{eV} \lesssim E \leqq 0.2 \mathrm{eV}$ the results are even quantitatively consistent. In this energy range several bands exist [see Fig. 4(c)] and thus there are more possible backscattering processes giving a larger scattering rate and thus a shorter MFP. Notice that lowering the mean free path does not necessarily mean reducing the conductance, because we also have more charge-carrying states.

Around the band edges at $E=-0.09 \mathrm{eV}$ the MFP calculated with FGR drops sharply whereas the values obtained with the GF and Kubo methods drop more slowly. This difference is caused by the relatively large disorder strength $\Delta \varepsilon=0.4 \mathrm{eV}$, which broadens the DOS and smears out the sharp features. For smaller disorder strengths the GF results 

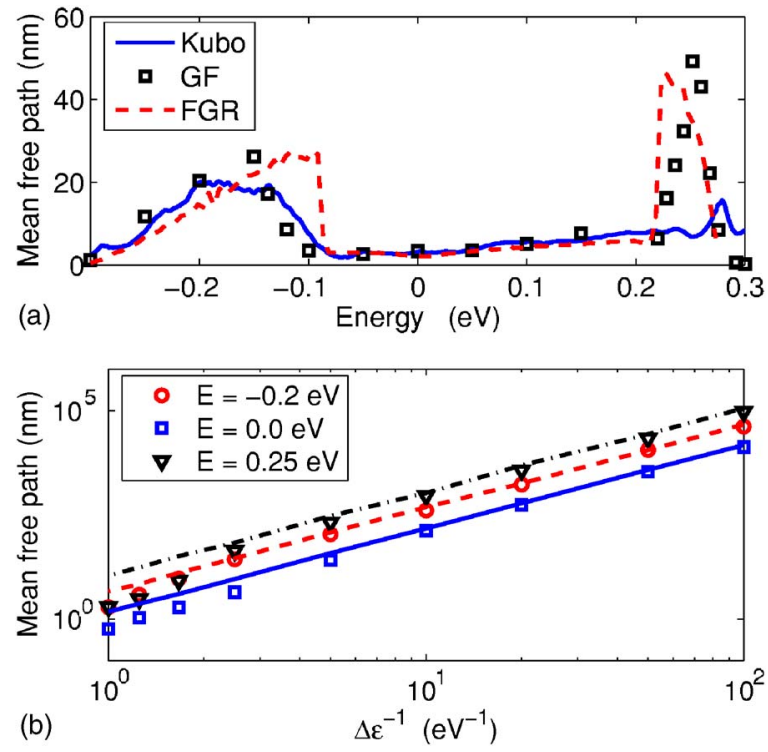

FIG. 6. (Color online). (a): mean free path $l_{e}$ vs energy $E-E_{F}$ for $\Delta \varepsilon=0.4 \mathrm{eV}$. The solid line is obtained with the Kubo method, and the GF results are marked by squares while the dashed line shows the FGR results. GF results are average values for 200 different samples while the Kubo results are mean values of 10 different samples. (b): scaling of $l_{e}$ vs $1 / \Delta \varepsilon$ shown on a logarithmic scale. Circles, squares, and triangles are calculated with GF while the lines are obtained using FGR at the same energies.

resemble the FGR values more, which is illustrated in the lower panel in Fig. 6. The figure shows, on a logarithmic scale, the MFP versus inverse disorder strength, $1 / \Delta \varepsilon$, at three different energies. The points are obtained with the GF method, while the lines are obtained using FGR. For weak disorder, the GF results scales as $l_{e} \propto(1 / \Delta \varepsilon)^{2}$ in accordance with FGR, whereas the GF results for strong disordered systems deviate from the $(1 / \Delta \varepsilon)^{2}$ dependence. In this regime the first-order perturbation applied in FGR does not fully suffices and reliable results must be obtained with more elaborate approaches such as the GF method.

Around $0.24 \mathrm{eV}$, the Kubo method fails to find the pronounced peak obtained with both the GF method and FGR. This difference is probably caused by two effects. The first reason is again a broadened DOS, since the GF results show a similar deviation from the FGR values as seen around $E$ $=-0.09 \mathrm{eV}$. The second and more important reason for the differences is due to numerical inaccuracies in calculating the density of states in the Kubo method. The inaccuracies are especially important around van Hove singularities at the subband edges. $E=0.24 \mathrm{eV}$ marks the band edge for the two degenerate bands, and due to the finiteness of the system considered in the Kubo calculation, the van Hove peaks in the DOS will unavoidably have decaying tails at larger energies. This implies that the calculated density of states will be too large causing $\mathcal{D}(E, t)$ and thus $l_{e}$ to be correspondingly smaller. The energy separation between the two subband edges around $E=0.24 \mathrm{eV}$ is only $\sim 0.05 \mathrm{eV}$, which is less than $0.3 \%$ of the total bandwidth, $W \approx 20 \mathrm{eV}$. This makes it numerically difficult to resolve the detailed features with the continued fraction technique used by the Kubo method.
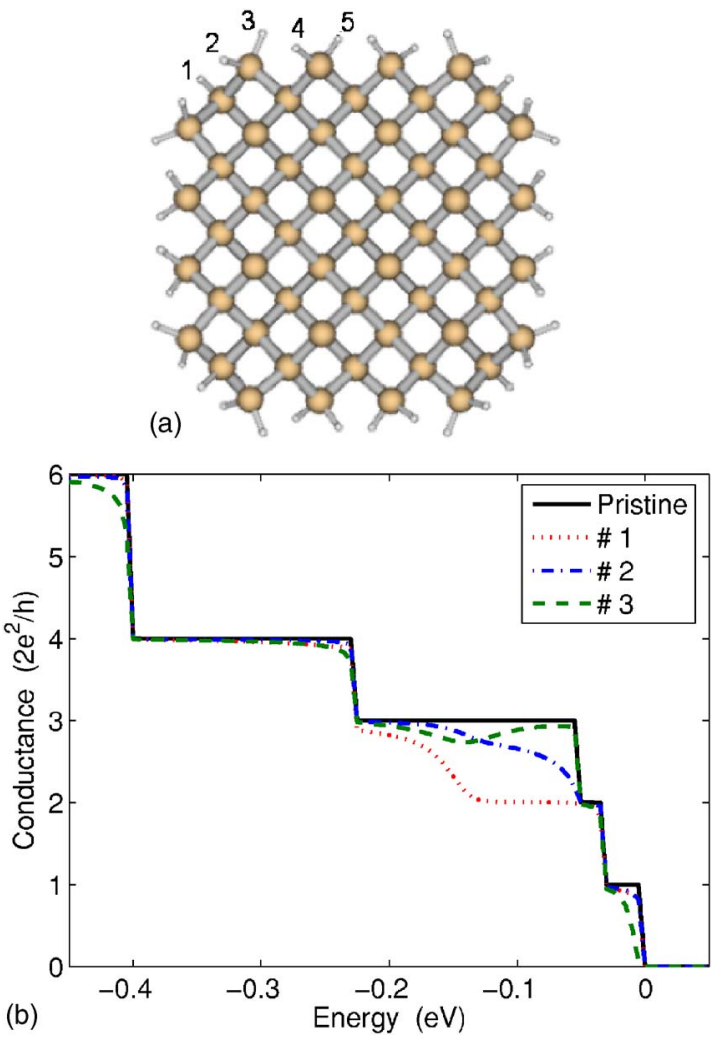

FIG. 7. (Color online) (a): cross section of the H-passivated wire. (a): energy-dependent conductance of a pristine wire and of wires containing a single vacancy of number 1-3.

\section{B. Passivated wires}

The surface-reconstructed SiNW's are both physically and technologically exciting, but probably also very fragile objects, since small changes in the surface such as defects or adatoms presumably will change the performance drastically. Moreover, the wires produced are most often surface passivated by either $\mathrm{SiO}_{x}$ or hydrogen, and the focus in this section will be on such wires. The passivated wires are semiconducting, often with a direct band gap that increases for small diameters. ${ }^{9,19,45}$ The simplest way to model surfacepassivated wires is to add hydrogen atoms to the surface such that all the Si dangling bonds are passivated. Such wires resemble qualitatively those reported by Ma et al. ${ }^{9}$

Figure 7 shows the cross section of the wire (a). The unit cell contains $57 \mathrm{Si}$ atoms and $36 \mathrm{H}$ atoms labeled with a number from 1 to 36 as indicated in Fig. 7(a). The band gap is found to be $2.84 \mathrm{eV}$. ${ }^{46}$

To investigate the influence of surface defects we introduce hydrogen vacancies. The vacancies are labeled correspondingly to the removed hydrogen atoms. Note that due to symmetry, there are only five topologically different vacancies. The conductances of wires with only a single vacancy, corresponding to one of the $\mathrm{H}$ atoms $1-3$ being removed, are shown in Fig. 7(b) for energies close to the valence band edge $(E=0 \mathrm{eV})$. Clearly, vacancies 1 and 2 scatter the most, the reason probably being that the wave function (for the pristine wire) has a pronounced larger weight on these $\mathrm{H}$ atoms. Notice that, for energies $-0.15 \mathrm{eV}<E<-0.05 \mathrm{eV}$, 


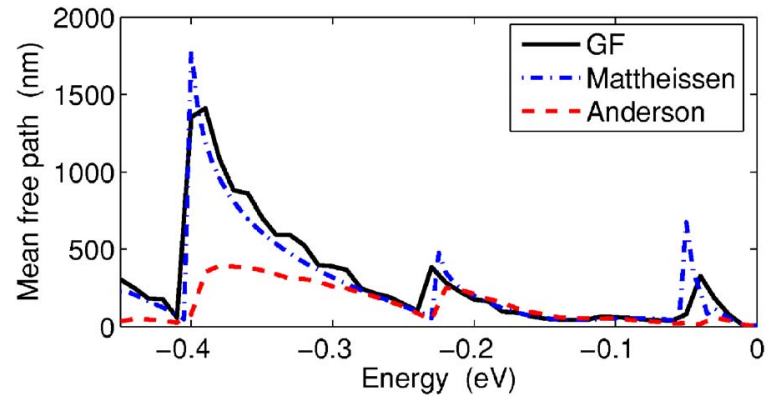

FIG. 8. (Color online) Mean free path vs energy for a concentration of vacancies corresponding to $\left\langle d_{H}\right\rangle=5.6 \mathrm{~nm}$. The solid black line corresponds to wires containing all possible vacancies. The dash-dotted blue line is obtained using Matthiessen's rule for single-scattering events and the dashed red curve corresponds to a wire with Anderson disorder $(\Delta \varepsilon=1.3 \mathrm{eV})$.

one channel is almost completely closed by vacancy 1 . Vacancies 3-5 give almost the same conductances.

We model a wire with randomly missing hydrogen atoms by performing a SIESTA calculation for each possible vacancy position (one of the $36 \mathrm{H}$ atoms is removed) and adding pieces from the different calculations. We measure the vacancy concentration by the average distance $\left\langle d_{H}\right\rangle$ in the wire direction between two vacancies. Each unit cell can only contain one vacancy, thus setting a lower limit for $\left\langle d_{H}\right\rangle$ at the unit cell length, $a=0.56 \mathrm{~nm}$. The MFP for $\left\langle d_{H}\right\rangle=5.6 \mathrm{~nm}$ calculated with the GF method is shown in Fig. 8 (solid line), revealing a strong energy dependence. In the interval $-0.15 \mathrm{eV}<E<-0.05 \mathrm{eV}$, we find $l_{e} \sim 50 \mathrm{~nm}$, while for energies around $-0.35 \mathrm{eV}$ the MFP in on the order of $1 \mu \mathrm{m}$. Comparing with estimated phonon scattering MFP's of more than $500 \mathrm{~nm},{ }^{16}$ the application of the elastic scattering model applied in this work is justified for most of the energies. Moreover, at several energies the vacancy scattering might be the dominant even at room temperature. However, around the peak at $E=-0.4 \mathrm{eV}$ where the calculated MFP's exceeds $1 \mu \mathrm{m}$, other scattering sources are likely to dominate.

Assuming that all bands at a given energy have the same reflection probability, $R_{i}(E)=\left[T_{0}(E)-T_{i}(E)\right] / T_{0}(E)$, where $T_{0}$ is the total transmission of a pristine wire and $T_{i}$ is the total transmission through a wire containing a single vacancy with number $i$ [shown in Fig. 7], the MFP in a wire with only vacancies of type $i$ can be estimated as $l_{e}^{(i)}(E)=\left\langle d_{H}^{(i)}\right\rangle / R_{i}(E)$, where $\left\langle d_{H}^{(i)}\right\rangle$ is the average distance between vacancies of type $i$. The total MFP can be estimated using Matthiessen's rule, such that $\tilde{l}_{e}^{-1}=\Sigma_{i}\left(l_{e}^{(i)}\right)^{-1}$, and the result is shown in Fig. 8 as the dash-dotted line. It is evident that the simple estimate, which ignores interference effects between successive scatterers, accurately reproduces the GF results found by sample averaging over vacancy configurations.

The Kubo method requires a Hamiltonian describing a wire that is longer than the largest mean free path in order to avoid boundary effects. As seen from Fig. 8 we therefore need a wire of length $L>1500 \mathrm{~nm}$ consisting of more than 3000 unit cells and thus $N>8 \times 10^{5}$ orbitals. In our current implementation this causes computer memory problems and we have not been able to obtain reliable results with the Kubo method for the vacancy scattering.

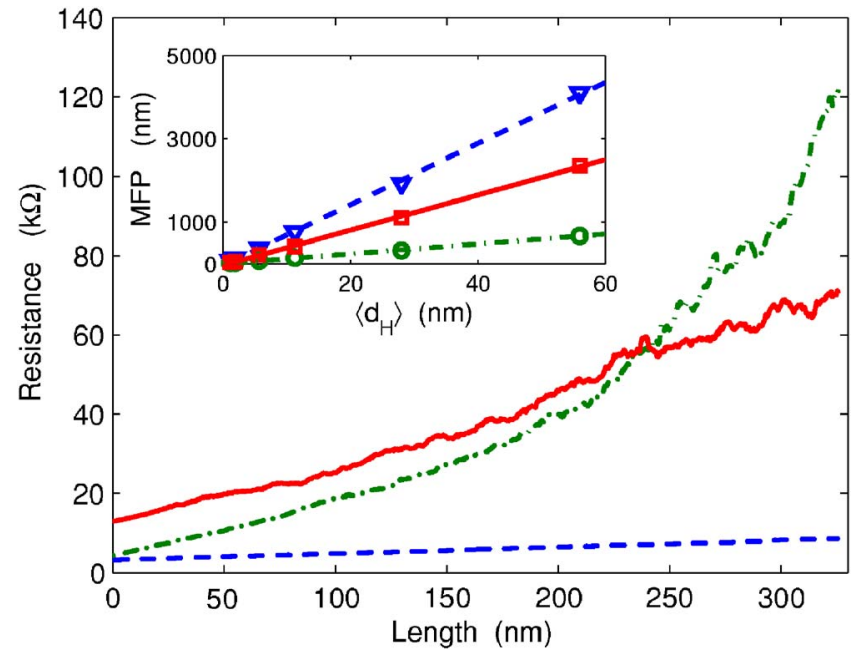

FIG. 9. (Color online) Length-dependent resistance at the energies $E=-0.3 \mathrm{eV}$ (dashed blue line), $E=-0.15 \mathrm{eV}$ (dash-dotted green line), and $E=-0.03 \mathrm{eV}$ (solid red line). The average distance between vacancies is $\left\langle d_{H}\right\rangle=2.8 \mathrm{~nm}$. The inset shows the scaling of $l_{e}$ vs average distance $\left\langle d_{H}\right\rangle$ between vacancies at the same three energies as above.

We next examine whether the vacancies effectively can be modeled by adding Anderson disorder. The calculated MFP for a surface disordered wire-i.e., with no vacancies but rather on-site disorder at all orbitals at the surface $\mathrm{Si}$ atoms - is shown in Fig. 8 (dashed red line).

For a disorder strength $\Delta \varepsilon=1.3 \mathrm{eV}$ the Anderson model fits the vacancy results at energies $E>-0.35 \mathrm{eV}$, although the peak around $E=-0.05 \mathrm{eV}$ is much less pronounced. The small shift of the peaks around $E=-0.23 \mathrm{eV}$ and $E=$ $-0.05 \mathrm{eV}$ is due to a broadened DOS in the Andersondisordered wires. For energies below $-0.35 \mathrm{eV}$, the Anderson model deviates significantly from the vacancy results. The pronounced peak in the MFP is not found in the Anderson model which gives almost constant values up to a factor of 3 lower than the vacancy results. Besides a broadened DOS, the differences probably arise because the Anderson model is a too simple model, not capturing all the physics. Since the Anderson disorder only reproduces the vacancy results at some energies, we conclude that the effects of vacancies cannot accurately be modeled by simple on-site disorder. Moreover, the value of the disorder strength, $\Delta \varepsilon$, has no clear connection to an actual vacancy concentration.

Figure 9 shows the length-dependent resistance for three different energies at vacancy concentration corresponding to $\left\langle d_{H}\right\rangle=2.8 \mathrm{~nm}$. For length $L<200 \mathrm{~nm}$ the resistance increases linearly at all energies, with the slope determining the MFP. For the curves in the figure a linear regression fit is performed for the initial part of the curves with $L<50 \mathrm{~nm}$ and the MFP is extracted using the relation $R(L)=R_{0}+R_{0} L / l_{e}$. For the curves in the figure we obtain a MFP of $199 \mathrm{~nm}$, $27 \mathrm{~nm}$, and $39 \mathrm{~nm}$ at the energies $-0.3 \mathrm{eV},-0.15 \mathrm{eV}$, and $-0.03 \mathrm{eV}$, respectively. For other vacancy concentrations and other energies the linear fit should be performed over another length range to ensure that it is confined to the linear part of the $R$ vs $L$ curve. Note that at $E=-0.03 \mathrm{eV}$ there is only one 
conducting channel [cf. Fig. 7(b)] and the contact resistance is thus $R_{0}=h / 2 e^{2}$. The inset shows the scaling of $l_{e}$ vs $\left\langle d_{H}\right\rangle$ at the same three energies as in the main figure. The points are calculated with the GF method and the lines are linear fits, clearly revealing a linear relationship between the MFP and the average intervacancy distance. For length $L>200 \mathrm{~nm}$ the resistance corresponding to $E=-0.15 \mathrm{eV}$ (dash-dotted green line) starts to increase exponentially, thus entering the localization regime. The localization length at this energy is $\xi$ $=110 \mathrm{~nm}$-i.e., approximately 4 times longer than the MFP. Although there are only three channels at $E=-0.15 \mathrm{eV}$, the results are roughly in agreement with the general rule $\xi$ $\sim N l_{e}$, where $N$ is the number of conducting channels. Within the energy interval $-0.225 \mathrm{eV}<E<-0.06 \mathrm{eV}$ where $N=3$ we find that the ratio $\xi / l_{e}$ varies between 3 at high energies and 5.6 at lower energies. The localization length can thus within a factor of 2 also be estimated from Matthiessen's rule.

\section{DISCUSSION}

In this paper we have studied electronic transport in SiNW's and calculated the influence of disorder on the mean free path. Our model is subject to a number of limitations and approximations. We apply a single-electron model and consider the linear response regime. Moreover, the minimal basis set may limit the accuracy. Also the spin-orbit coupling is not included which is necessary to describe the details around the valence band edge in bulk Si. Compared to the experimentally realized SiNW's, the wires considered here are quite thin although comparable to the wires reported in Ref. 9. The SiNW structures used in this paper have rounded, rather than perfectly square angles. It has been proposed in the literature ${ }^{47-49}$ that smooth angles would be favored during the growth process with respect to the sharp angles that naturally arise from the square symmetry of the [100] cleavage plane. The topic has been discussed at some details elsewhere ${ }^{49}$ for surface-reconstructed wires, concluding that at nanometric diameters no clear difference emerges. At the same time the electronic structure seems to be only marginally affected. Since all calculations in this work are performed on relaxed structures fully based on ab initio calculations without any use of fitting parameters, we expect, in spite of all the limitations, to capture the correct trends in the transport characteristics.

\section{A. Methods}

Two numerical methods were applied and compared to each other: a real-space Kubo method and a recursive Green's-function method. The two approaches each have their advantages: In calculating the MFP at many energies, the Kubo method is advantageous, since the diffusion is readily found at many energies in a single calculation, whereas the GF method requires a full calculation at each energy. If the focus is on a few energies but many different disorders, the GF method is the preferred choice. For metallic systems, where one mainly is interested in the properties around the Fermi level, the GF method thus seems to be the method of choice-the parallel computation of many ener- gies in the Kubo method is not needed. The Kubo method seems more applicable to semiconducting systems, since it is physically possible to move the Fermi level with a gate voltage, thereby scanning several energies.

The Kubo method requires a Hamiltonian describing a wire that is longer than the largest MFP in the considered energy range. For the weakly disordered wires with long MFP's we need wires of length $L \geqslant 1 \mu \mathrm{m}$ containing more than $10^{5}$ atoms, resulting in very large matrices. In our current implementation this causes memory problems and the Kubo method failed to converge for the H-passivated SiNW's. The GF method, on the other hand, involves only calculations with the small subcell Hamiltonians and it suffices to consider wires grown to a length $L \approx 50 \mathrm{~nm}$ to get an accurate estimate of the initially linear resistance versus length curve (cf. Fig. 9). Moreover, it proved to be numerically difficult to resolve the detailed features in the energy spectrum with the Kubo method, which led to erroneous results near band edges. The difficulties arise because the Kubo method does not take any semi-infinite periodic leads into account as in the GF method. There, the DOS is readily calculated to arbitrary accuracy from the surface Green's function $\mathbf{G}_{L}^{0}$ by using the periodic structure of the leads. However, the absence of periodic leads in the Kubo method can also be a great advantage since it allows one to study nonperiodic systems such as incommensurable multiwalled carbon nanotubes. ${ }^{24}$

The growth procedure in the GF method involves inversions of the relatively small matrices describing the subcells. For thin wires as those considered in this work, with $N$ $\sim 250$ orbitals in each unit cell, the inversion step is not a critical issue. However, for thicker wires the number of orbitals increases quadratically, and due to as $O\left(N^{3}\right)$ scaling of the inversion step, the whole procedure scales as $O\left(d^{6}\right)$, with $d$ being the wire diameter. On contrary, the Kubo method scales linearly with the number of orbitals and, thus, as $O\left(d^{2}\right)$.

For systems with a periodic structure, as the SiNW's considered here, we generally find the GF method to be the preferred choice, given that a rough energy resolution is sufficient. Thicker wires with relatively short MFP's favor the use of the Kubo approach.

\section{B. Results}

In unpassivated, surface-reconstructed wires, Anderson disorder was added to the surface atoms, affecting the transport properties significantly. Disorder in the bulk had, on the other hand, no significant influence.

In hydrogen-passivated wires surface disorder was introduced by randomly removed hydrogen atoms. We find that it suffices to consider single-scattering events and adding the individually calculated MFP's to an effective MFP by applying Matthiessen's rule. Using the rule $\xi \sim N l_{e}$, where $N$ is the number of conducting channels, the localization length can be estimated within a factor of 2. However, an accurate determination of $\xi$ seems to require full calculations on long wires with many randomly placed $\mathrm{H}$ vacancies as opposed to only considering single-scattering events. It was further 

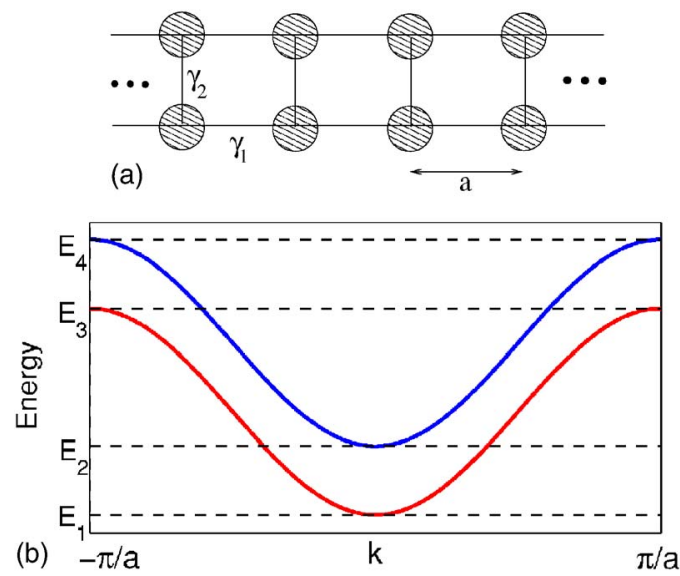

FIG. 10. (Color online) (a): model system. We consider only nearest-neighbor interaction with the tight-binding parameters $\gamma_{1}$ and $\gamma_{2}$ corresponding to hopping in the chain direction and in perpendicular direction, respectively. The on-site energy is $\varepsilon_{0}$. (b): band structure for the two bands separately. Notice the definition of the energies $E_{1}-E_{4}$.

shown that an attempt to model the vacancies with an effective Anderson disorder gave satisfactory values for energies close to the valence band edge but failed to reproduce the vacancy results at lower energies.

The MFP was shown to be strongly energy dependent, and for relatively long wires the resistance can be changed by orders of magnitude within a $0.1-\mathrm{eV}$ shift of the Fermi energy, thus causing a transition from the diffusive (Ohmic) regime to the localization regime. The strong energy dependence might be utilized in sensor applications where the presence of a single virus acts as a local gate shifting the Fermi energy. ${ }^{8}$ This could possibly cause a transition from the Ohmic to the localization regime, thus changing the resistance of the wire dramatically. However, more careful work has to be done before firm conclusions can be stated.

For relatively strong disordered wires, the MFP is well below $500 \mathrm{~nm}$ for a large energy range. Compared to estimated phonon scattering MFP's of more than $500 \mathrm{~nm},{ }^{16}$ the elastic scattering model applied in this work is justified. Comparing the results obtained in this work with the estimated long phonon MFP we suggest that impurity and defect scattering could be the dominant scattering source even at room temperature.

\section{ACKNOWLEDGMENTS}

We thank H. Smith and N. Lorente for discussions and the Danish Center for Scientific Computing (DCSC) for providing computer resources. T.M. thanks the Oticon Foundation for financial support. R.R. acknowledge financial support of the Ministerio de Educación y Ciencia through the Juan de la Cierva programme.

\section{APPENDIX: SIMPLE DOUBLE-CHAIN MODEL}

In this appendix we illustrate the difficulties in calculating the group velocity in the Kubo method. The problem is most
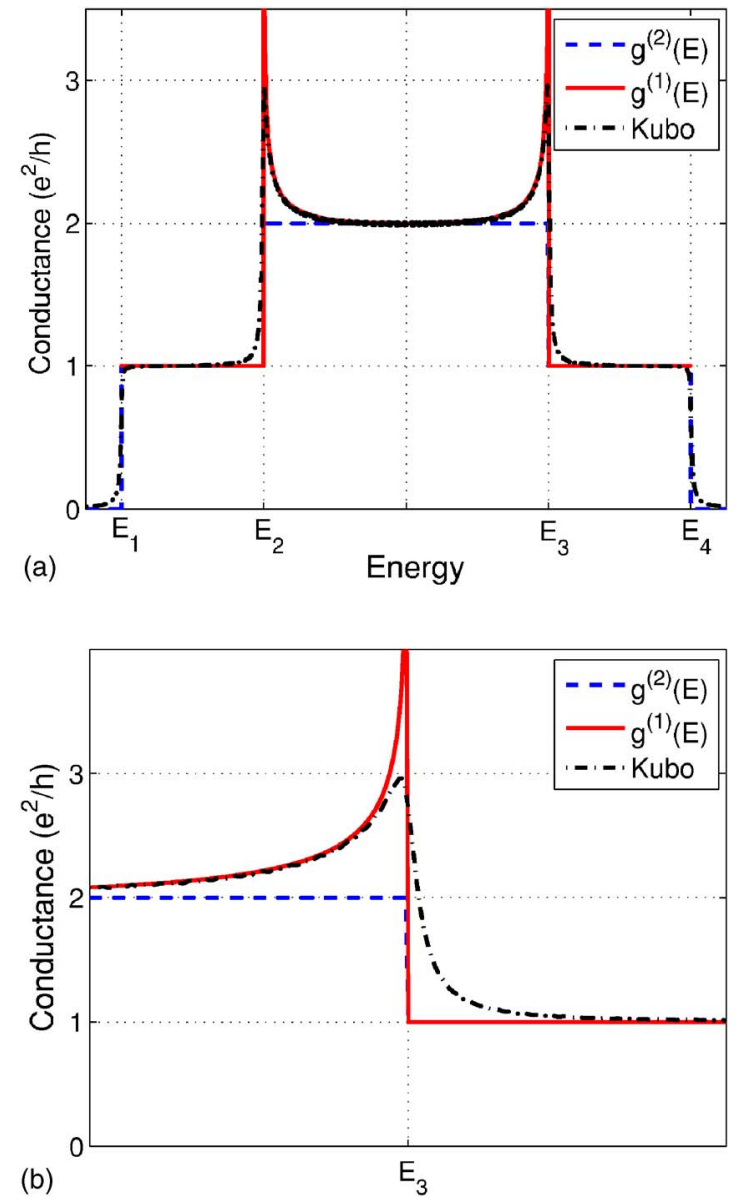

FIG. 11. (Color online). (a): the numerically calculated conductance (dash-dotted black line) is plotted together with the two analytical results $g^{(1)}(E)$, Eq. (A6) (dashed blue line) and $g^{(2)}(E)$, Eq. (A7) (solid red line). (b): zoom-in around $E=E_{3}$, showing that $g^{(1)}(E)$ closely resembles the numerical result.

pronounced near band edges when more bands are present.

We consider a model consisting of two infinite parallel chains as shown in Fig. 10 (a). Only nearest-neighbor interactions are taken into account. The tight-binding parameters are $\gamma_{1}$ and $\gamma_{2}$ for hopping between and along the chains, respectively. The distance between two atoms in the same chain is called $a$. In the Fourier domain the Hamiltonian is

$$
\mathbf{H}(k)=\left(\begin{array}{cc}
\varepsilon_{0}+2 \gamma_{1} \cos (k a) & \gamma_{2} \\
\gamma_{2} & \varepsilon_{0}+2 \gamma_{1} \cos (k a)
\end{array}\right),
$$

where $\varepsilon_{0}$ is the on-site energy. The eigenvalues are

$$
E_{ \pm}(k)=\varepsilon_{0}+2 \gamma_{1} \cos (k a) \pm \gamma_{2},
$$

and the band structure is shown in Fig. 10 (b) with $\varepsilon_{0}=0$ and $\gamma_{1}=\gamma_{2}$. The density of states and the group velocities for the two bands are found as $n_{ \pm}=1 /(2 \pi)\left|\partial E_{ \pm}(k) / \partial k\right|^{-1}$ and $v_{ \pm}$ $=1 /(\hbar) \partial E_{ \pm}(k) / \partial k$.

The problems in the Kubo method are clearest illustrated by calculating the conductance. Following Ref. 23 we start out from an Einstein-like conductivity $\sigma(E, t)$ $=e^{2} n(E) \mathcal{D}(E, t)$, where $n(E)$ is the total electronic density of 
states. The conductance of a sample of length $L$ is found as $g(E)=\sigma(E, \tau) / L$, where $\tau$ is the time is takes a wave packet to spread out by an amount equal to $L$-i.e., implicitly given by the relation $L=\sqrt{\mathcal{X}^{2}(E, \tau)}$, where $\mathcal{X}^{2}(E, t)=t \mathcal{D}(E, t)$. At present we consider only a pristine system where the electron propagation is ballistic with $\mathcal{X}^{2}(E, t)=v^{2}(E) t^{2}$, and the conductance thus becomes

$$
g(E)=e^{2} n(E) v(E) .
$$

We calculate the diffusion coefficient as given by Eq. (1). In the energy interval $E \in\left[E_{2} ; E_{3}\right]$ there are two bands and the $\delta(E-\mathbf{H})$ projects the states in the trace into a linear combination of the eigenstates from the two bands. We can therefore rewrite Eq. (1) as

$$
\mathcal{D}(E, t)=\frac{1}{t} \frac{n_{+}(E) X_{+}^{2}(E)+n_{-}(E) X_{-}^{2}(E)}{n_{+}(E)+n_{-}(E)},
$$

where $X_{ \pm}^{2}(E)=\left[v_{ \pm}(E) t\right]^{2}$ is the spread of wave packets belonging to each band. Defining an effective velocity as (for clarity we omit the explicit energy dependence)

$$
v_{\mathrm{eff}}^{(1)}=\sqrt{\frac{n_{+} v_{+}^{2}+n_{-} v_{-}^{2}}{n_{+}+n_{-}}},
$$

the diffusion coefficient becomes $\mathcal{D}(E, t)=\left(v_{\text {eff }}^{(1)}\right)^{2} / t$. Using Eq. (A3) the conductance becomes

$$
g^{(1)}(E)=e^{2}\left(n_{+}+n_{-}\right) \sqrt{\frac{n_{+} v_{+}^{2}+n_{-} v_{-}^{2}}{n_{+}+n_{-}}},
$$

in disagreement with the correct result. The conductance should rather be

$$
g^{(2)}(E)=e^{2}\left[n_{+} v_{+}+n_{-} v_{-}\right],
$$

which is obtained if we instead of the effective velocity, $v_{\mathrm{eff}}^{(1)}$ in Eq. (A5) use the average velocity

$$
v_{\text {eff }}^{(2)}=\frac{n_{+} v_{+}+n_{-} v_{-}}{n_{+}+n_{-}} .
$$

Figure 11 (a) shows the two analytical expressions for the conductances $g^{(1)}(E)$ (solid red line) and $g^{(2)}(E)$ (dashed blue line) together with the numerical result (dash-dotted black line) obtained using the Kubo method and time propagation; the lower panel shows an expanded view around $E=E_{3}$.

It is evident that $g^{(1)}(E)$, Eq. (A6), resembles the numerical result very much. Especially we find the same peaks in the conductance around the band edges. The tail in the Kubo conductance is due to the finiteness of the system and reflects the imaginary energy $i \eta$ used in the continued fraction technique. Rewriting the effective velocity (A5) as

$$
v_{\mathrm{eff}}^{(1)}=\frac{\sqrt{\left(n_{+} v_{+}+n_{-} v_{-}\right)^{2}+n_{+} n_{-}\left(v_{+}-v_{-}\right)^{2}}}{n_{+}+n_{-}},
$$

we see that the reason why we observe peaks in the conductance is a mixing of the two densities of states given by the second term in the numerator. This mixing is an inherent failure in the numerical calculation of the diffusion coefficient.
${ }^{1}$ Y. Cui and C. M. Lieber, Science 291, 851 (2001).

${ }^{2}$ M. S. Gudiksen, L. J. Lauhon, J. Wang, D. C. Smith, and C. M. Lieber, Nature (London) 415, 617 (2002).

${ }^{3}$ L. Samuelson, Mater. Today 6, 22 (2003).

${ }^{4}$ Y. Cui, D. Wang, W. U. Wang, and C. M. Lieber, Nano Lett. 3, 149 (2003).

${ }^{5}$ L. Samuelson et al., Physica E (Amsterdam) 25, 313 (2004).

${ }^{6}$ Y. Wu, C. Yang, W. Lu, and C. M. Lieber, Nature (London) 430, 61 (2004).

${ }^{7}$ Y. Wu, Y. Cui, L. Huynh, C. Barrelet, D. Bell, and C. Lieber, Nano Lett. 4, 433 (2004).

${ }^{8}$ F. Patolsky and C. M. Lieber, Mater. Today 8, 20 (2005).

${ }^{9}$ D. D. D. Ma, C. S. Lee, F. K. Au, S. T. Tong, and S. T. Lee, Science 299, 1874 (2003).

${ }^{10}$ J. D. Holmes, K. Johnston, R. C. Doty, and B. A. Korgel, Science 287, 1471 (2000).

${ }^{11}$ Y. Cui, L. J. Lauhon, M. S. Gudiksen, J. Wang, and C. M. Lieber, Appl. Phys. Lett. 78, 2214 (2001).

${ }^{12}$ R. Rurali and N. Lorente, Phys. Rev. Lett. 94, 026805 (2005).

${ }^{13}$ A. K. Singh, V. Kumar, R. Note, and Y. Kawazoe, Nano Lett. 5, 2302 (2005).

${ }^{14}$ M. V. Fernandez-Serra, C. Adessi, and X. Blase, Phys. Rev. Lett.
96, 166805 (2006).

${ }^{15}$ T. Vo, A. J. Williamson, and G. Galli, Phys. Rev. B 74, 045116 (2006).

${ }^{16}$ W. Lu, J. Xiang, B. P. Timko, Y. Wu, and C. M. Lieber, Proc. Natl. Acad. Sci. U.S.A. 102, 10046 (2005).

${ }^{17}$ K. K. Das and A. Mizel, J. Phys.: Condens. Matter 17, 6675 (2005).

${ }^{18}$ V. S. Sundaram and A. Mizel, J. Phys.: Condens. Matter 16, 4697 (2004).

${ }^{19}$ Y. Zheng, C. Riva, R. Lake, K. Alam, T. B. Boykin, and G. Klimeck, IEEE Trans. Electron Devices 52, 1097 (2005).

${ }^{20}$ S. Roche, Phys. Rev. B 59, 2284 (1999).

${ }^{21}$ S. Roche and D. Mayou, Phys. Rev. Lett. 79, 2518 (1997).

${ }^{22}$ D. Mayou, Phys. Rev. Lett. 85, 1290 (2000).

${ }^{23}$ S. Roche and R. Saito, Phys. Rev. Lett. 87, 246803 (2001).

${ }^{24}$ F. Triozon, S. Roche, A. Rubio, and D. Mayou, Phys. Rev. B 69, 121410(R) (2004).

${ }^{25}$ S. Latil, S. Roche, D. Mayou, and J.-C. Charlier, Phys. Rev. Lett. 92, 256805 (2004).

${ }^{26}$ S. Roche, J. Jiang, F. Triozon, and R. Saito, Phys. Rev. B 72, 113410 (2005).

${ }^{27}$ S. Roche, J. Jiang, F. Triozon, and R. Saito, Phys. Rev. Lett. 95, 
076803 (2005)

${ }^{28}$ S. Latil, S. Roche, and J.-C. Charlier, Nano Lett. 5, 2216 (2005).

${ }^{29}$ R. Kubo, J. Phys. Soc. Jpn. 12, 570 (1957).

${ }^{30}$ D. Greenwood, Proc. Phys. Soc. London 71, 585 (1958).

${ }^{31}$ C. T. White and T. N. Todorov, Nature (London) 393, 240 (1998).

${ }^{32}$ F. Triozon, J. Vidal, R. Mosseri, and D. Mayou, Phys. Rev. B 65, 220202(R) (2002).

${ }^{33}$ R. Haydock, V. Heine, and M. Kelly, J. Phys. C 5, 2845 (1972).

${ }^{34}$ T. N. Todorov, Phys. Rev. B 54, 5801 (1996).

${ }^{35}$ M. Lopez-Sancho, J. Lopez-Sancho, and J. Rubio, J. Phys. F: Met. Phys. 14, 1205 (1984).

${ }^{36}$ S. Datta, Electronic Transport in Mesoscopic Systems (Cambridge University Press, Cambridge, England, 1995).

${ }^{37}$ B. Kramer and A. MacKinnon, Rep. Prog. Phys. 56, 1469 (1993).

${ }^{38}$ J. M. Soler, E. Artacho, J. D. Gale, A. García, J. Junquera, P. Ordejón, and D. Sánchez-Portal, J. Phys.: Condens. Matter 14, 2745 (2002).

${ }^{39}$ E. Artacho, D. Sánchez-Portal, P. Ordejón, A. García, and J. M. Soler, Phys. Status Solidi B 215, 809 (1999).

${ }^{40}$ N. Troullier and J. L. Martins, Phys. Rev. B 43, 1993 (1991).
${ }^{41}$ J. P. Perdew, K. Burke, and M. Ernzerhof, Phys. Rev. Lett. 77, 3865 (1996).

${ }^{42}$ H. J. Monkhorst and J. D. Pack, Phys. Rev. B 8, 5747 (1973).

${ }^{43}$ J. Zhong and G. M. Stocks, Nano Lett. 6, 128 (2006).

${ }^{44} \mathcal{D}(E, t)$ is calculated slightly above the Fermi energy in order to avoid the numerical difficulties at $E=E_{F}$, where a band edge occur.

${ }^{45}$ X. Zhao, C. M. Wei, L. Yang, and M. Y. Chou, Phys. Rev. Lett. 92, 236805 (2004).

${ }^{46}$ The calculated band gap is larger than found in Ref. 50 for a similar structure using a plane-wave DFT method. The band gap reduces to $\sim 1.66 \mathrm{eV}$ when using a double- $\zeta$ polarized basis set, in qualitiative agreement with the plane-wave results of Ref. 50 (the residual difference must be attributed to the slight differences in the wire structures).

${ }^{47}$ S. Ismail-Beigi and T. Arias, Phys. Rev. B 57, 11923 (1998).

${ }^{48}$ Y. Zhao and B. I. Yakobson, Phys. Rev. Lett. 91, 035501 (2003).

${ }^{49}$ R. Rurali and N. Lorente, Nanotechnology 16, S250 (2005).

${ }^{50}$ A. K. Singh, V. Kumar, R. Note, and Y. Kawazoe, Nano Lett. 6, 920 (2006). 